\title{
MENOMETRORRHAGIA IN MAGNETIC RESONANCE IMAGING OPERATORS WITH COPPER INTRAUTERINE CONTRACEPTIVE DEVICES (IUDS): A CASE REPORT
}

\section{FABRIZIOMARIA GOBBA ${ }^{1}$, NADIA BIANCHI ${ }^{2}$, PAOLO VERGA ${ }^{3}$, GIAN MARCO CONTESSA ${ }^{4}$, and PAOLO ROSSI ${ }^{4}$}

${ }^{1}$ University of Modena and Reggio Emilia, Modena, Italy

Department of Public Health Sciences

${ }^{2}$ Regional Health Service, Varese, Italy

Radioprotection Unit

${ }^{3}$ S. Carlo Borromeo Hospital, Milano, Italy

${ }^{4}$ Italian Worker's Compensation Authority (INAIL), Rome, Italy

\begin{abstract}
The paper describes the cases of 3 female health operators with implanted copper IUDs, developing menometrorrhagia some months after an increase of the working time in a Magnetic Resonance Imaging (MRI) Unit (1.5 T), that progressively disappeared when the previous organization, involving discontinuous work shifts at MRI, was re-established. No known factors possibly related to menometrorrhagia were evidenced in the 3 operators, supporting the hypothesis of a role of the exposure to the electromagnetic fields (EMF) induced by the MRI system in symptoms induction. The possible mechanism remains unsettled, but menometrorrhagia might be triggered by a phlogistic stimulus caused by EMF, possibly the lowfrequency currents induced in the wires of the IUD during the movements of the operator inside the static magnetic field generated by the MRI permanent magnet. Until now, the problem of possible interactions between copper IUDs and EMF induced by MRI has been considered in patients undergoing imaging, but the possible risk in MRI Units operators has been largely neglected. To our knowledge, the occurrence of menometrorrhagia is not routinely checked in health surveillance of MRI operators, so these symptoms can pass unnoticed, especially if they are transitory. Therefore, underreporting is rather possible. The cases described here support the need for further research on this topic, especially considering the progressive diffusion of more powerful MRI scanners (3 T and more), and of the interventional magnetic resonance imaging, both potentially involving higher EMF exposures, and a large number of MRI female operators, possibly using IUDs. The possibility that MRI operators with implanted metallic IUDs can be included in the group of "workers at particular risk" according to the EU Directive 2004/40/EC should be considered.
\end{abstract}

Key words:

Static magnetic fields, Occupational exposure, Induced currents, Workers at particular risk, Menometrorrhagia

Received: August 30, 2011. Accepted October 28, 2011.

Address reprint request to F. Gobba, Cattedra di Medicina del Lavoro, Università di Modena e Reggio Emilia, Dipartimento di Scienze di Sanità Pubblica, Via Campi 287-41125 Modena, Italy (e-mail: fabriziomaria.gobba@unimore.it). 


\section{INTRODUCTION}

The possible safety risk related to Magnetic Resonance Imaging (MRI) in patients with implanted intrauterine contraceptive devices (IUDs) is a known problem [1]. In this context, the main types of IUD devices that can be distinguished are the metal-containing/metal-free intrauterine device (IUD), the fully metal-free hormone-containing intrauterine system (IUS), and the ESSURE, an insert made of stainless steel, in use in Europe since 2001 [2,3]. The main concern was related to the possibility that, in patients with metal-containing IUD or with ESSURE, an injury can be induced by heating, or movement of the device during scanning [4-6]. An additional concern was that the diagnostic accuracy of the image would be impaired by artifact created by the device [7]. For these reasons, in the past, the presence of IUD was considered a limit to MRI scanning, but the conclusion of more recent studies is that up to a magnetic field strength of $1.5 \mathrm{~T}$ there is no risk of any movement or dislodgement of both the IUD and the ESSURE, and that a possible temperature increase, if any, is within the physiologic range, at least if the IUDs are placed in fixed position inside the bore of the MRI system [4-6]. Accordingly, intrauterine contraceptive devices are currently considered safe for patients imaged with MR systems operating at $1.5 \mathrm{~T}$ or less [1,4-6,8].

At $3 \mathrm{~T}$ or above, only the metal-free IUDs and the IUS are considered MRI-safe, while metal-containing IUDs and the ESSURE have not yet been considered adequately evaluated [2], and the American Society for Testing and Materials and the Food and Drug Administration classified IUD as "MR conditional" [7,9].

The aforementioned evaluations substantially concern patients undergoing MRI for medical reasons. Nevertheless, an exposure to electromagnetic fields (EMF) occurs also in personnel working with, and around, the medical MR equipment, including radiology technicians, nurses, radiologists, anesthetists and surgeons, and also the maintenance and cleaning staff $[10,11]$. The presence of personnel during MRI scanning is usually limited to a few specific situations, as in some cardiological examinations in anesthetized patients, or assistance to children, but health personnel (and maintenance staff) at MRI Units are currently exposed to the static magnetic field (SMF) generated by the magnet. The motion of subjects along a field gradient, or the rotational motion while working in the MRI room can induce significant low-frequency currents inside the body, which has been estimated in some studies [10,11].

To our knowledge, the problem of implanted IUDs in MRI Unit personnel has so far been neglected, and no data is currently available on the possible risk related to EMF generated by the MRI equipment in these subjects. We hereby describe three cases of menometrorrhagia observed in female workers of an MRI unit.

\section{CASE PRESENTATION}

In the Radiology Department of a hospital in a Milan area (Lombardy Region, Italy), a 1.5 T MRI scanner has been in use since 2004. Among the female personnel of the Unit, six radiology technicians and 1 nurse, aged 23-36 years, have been engaged in MRI procedures. The usual activity of technicians and nurses was limited to the assistance to the patients, but as a rule they were not present during the MRI scanning. Accordingly, they were exposed to the SMF but, customarily, not to radiofrequency nor gradient time-varying magnetic fields related to the scanning. The operators moved randomly inside the SMF, often very close to the gantry. An exposure during MRI scanning was possible, but really uncommon, limited to occasional situations as in case of assistance to children.

In 2004 and 2005, the personnel of the Department was on duty in the MRI Unit based on a rotational system, one week per month. Work shifts in the MRI Unit usually lasted 8 hours per day, even though overtime was not exceptional, especially in the case of emergencies. 
In 2006, the work schedule was re-organized, and fullmonth shifts in the MRI Unit were adopted. In 2007, the one-week/month MRI shifts were re-established.

The whole personnel of the Radiology Department underwent a yearly medical examination by an occupational physician, as part of the program of health surveillance (HS) of the hospital staff. Apparently, until 2006, no remarkable health problems had been reported by the Radiology Department staff. During the examinations performed in 2006 (second half), in five out of the 7 MRI Unit female health professionals, menometrorrhagia (irregular or excessive bleeding during menstruation and/or between menstrual periods) was recorded. The symptom was unexpected, so medical records of all the female personnel of the MRI Unit were revised.

Three of the 5 subjects reporting menometrorrhagia, aged 33, 40 and 43 years old, had an implanted copper T3 IUD. According to the medical history, no previous gynecological disorders had been recorded, and the symptoms appeared after some weeks of full-month work in the MRI Unit. During the visit, the operators stated that the symptoms were persistent for about 6 months or more, without any significant variation over the time. None of these 3 subjects contacted a specialist in connection with the symptoms. The blood count was normal, and neither symptoms suggesting endocrine or systemic disorders, nor persistent bleeding, dyspareunia, dysmenorrhea, pelvic pain and vaginal discharge, were reported. No other investigations, such as pelvic ultrasound, were performed.

Menometrorrhagia was also reported by two MRI Unit healthcare professionals not using IUDs, both taking oral gestodene/ethinyl estradiol contraceptives. In one case, the worker was unsure regarding the period of appearance of the symptom, while in the other, menometrorrhagia had pre-existed for some years, and Polycystic Ovary Syndrome (POS) had previously been diagnosed in a gynecological examination.
In the other 2 women working in the MRI Unit no menometrorrhagia was reported.

Due to the observation of the appearance of menometrorrhagia, in the majority of the female MRI personnel, after an increase of time on duty at the Unit, a possible role of MRI-related electromagnetic fields (EMF) was hypothesized, and the problem was discussed with the hospital management. Even tough the actual role of EMF in the symptoms appearance was not established, a decision was taken to reduce the exposure; consequently the previous work organization of the Radiology Department (one week/month on duty in the MRI Unit) was restored.

In the following year, according to the HS recordings, menometrorrhagia slowly subsided in the 3 women bearing IUDs, while no significant change was noted by the two women taking oral contraceptives.

Increased menstrual bleeding, often with pain, is considered one of the most common problem of IUD use, especially during the first months after application, and it is the most common medical reason for removing IUDs: in clinical trials about $4 \%$ to $15 \%$ of women stop using IUDs for this reason within a year after insertion [3]. With regards to our case report, we have to observe that the workers had been using the IUDs for some years without any apparent bleeding. The symptoms started after a significant increase of the time spent working in the MRI Unit (continuous activity instead of 1 week/month, as previously), and gradually subsided after the newly introduced work system in the MRI Unit was discontinued (1 week/month).

Both other two workers (without IUDs) complaining of menometrorrhagia took gestodene/ethinyl estradiol oral contraceptives. Abnormal uterine bleeding is a common side effect of all forms of hormonal contraception [12]. Furthermore, at least in one case, POS was diagnosed, which could be responsible for the symptom [13]. According to the HS records, in both workers, menometrorrhagia remained substantially unmodified after re-establishing the previous "one week/month at MRI" work organization. 
So far, data on the possible effects induced by MRI in women with IUDs is mainly limited to an evaluation of the possible effects in patients undergoing MRI imaging for medical reasons, often using models $[4,7,9]$. Very few cases of pelvic discomfort, cramping and bleeding - while having magnetic resonance imaging of the pelvic area - have been described [4], but bleeding, or menometrorrhagia, following an MRI scan can pass unnoticed, especially in case of transitory symptoms, and, accordingly, underreporting is possible. Another aspect to be considered here is that the exposure to MRI-related fields is very different between patients and operators. In fact, health personnel members are rarely exposed to the fields generated by imaging (except in specific cases, as in cardiological examination in anesthetized patients or children assistance), but are currently exposed to the SMF related to the permanent magnet of the MRI Unit, and to the low-frequency currents induced by the movement of subjects inside the SMF [10]. Although the patients are exposed to the static field as well, the differences between the patients and MRI staff are relevant, e.g. in terms of movements (e.g. length, speed, position) and exposure time (patients are exposed for some minutes, while health personnel for several hours a day during the whole working week).

In the cases described here, menometrorrhagia appeared when the duty in the MRI Unit was extended to the whole month instead of one week/month; moreover the symptoms were contemporarily present in the 3 women with implanted IUD, and subsided following a reduction of the exposure due to duty reorganization, along with an absence of significant gynecological disorders recorded in their medical history. This data supports a possible link between the exposure to MRI-related EMF and the symptoms.

As excessive physical effort is among the possible causes of menometrorrhagia [14], the possibility that the symptoms were related to an increase of the work-load linked to the change of work-organization cannot be totally excluded, but we have to consider the fact that the re-organization of the work involved a change of activity (whole month in the MRI Unit, instead of 1 week/month) rather than in an increase in scope of the daily activity. Other common causes for menometrorrhagia were not identified from medical records [14].

The possible mechanism for MRI-related EMF bleeding induction can only be presumed. The copper IUD used by the operators consisted of a plastic core body, surrounded by copper wires [15]. Up to magnetic field strength of $1.5 \mathrm{~T}$, as in this case, literature data does not support the possibility of heating or movements of the IUD during scanning [1,4-6,8]. Nevertheless, so far (to our knowledge) studies have taken into account only the case of IUDs placed in fixed positions inside the bore. This situation is rather different from the case of MRI operators: copper is diamagnetic, and the movement inside the SMF can cause a magnetic flux inside the wires of the IUD, inducing lowfrequency currents depending on the strength, position, speed, etc. [15], and the interaction of the involved physical quantities with the living tissues can result in different outcomes. Especially in case of prolonged exposures, the direct influence of the induced currents to the tissues might be responsible for a phlogistic stimulus to the mucosa, leading to bleeding. This hypothesis may be applied to the three IUD-bearing women presented in this case report: when work in the MRI Unit was 1 week/month, the exposure was discontinued, and the stimulus was insufficient to cause menometrorrhagia (or the symptoms were so mild that they were not noticed), but when work in the MRI Unit was extended to last for the whole month, exposure was continued, and the phlogistic stimulus became sufficient to induce overt clinical symptoms.

Similar observations in MRI workers are very limited, but, according to our knowledge, the occurrence of menometrorrhagia is not routinely evaluated in the health surveillance of MRI operators, so these symptoms can pass unnoticed, especially if they are transitory. Therefore, underreporting is possible/likely. In any case, we have to observe that at least 
one other similar case has been described in the scientific literature. It was a woman who had been bearing a copper IUD for 4 years without complications. Then, two months after starting a new job with MRI machines, she reported pelvic discomfort and vaginal bleeding every two weeks [4]. The similarity to the cases described here is remarkable.

\section{CONCLUSIONS}

The reported observations of the appearance of menometrorrhagia in female operators with copper IUDs after an increase of the working time in an MRI Unit, that ceased to occur when exposure was reduced and discontinued, and the absence of other obvious factors, are coherent with the hypothesis of an adverse effect caused by the EMF fields induced by the MRI equipment. The description of at least one other case in the scientific literature, and the possibility of underreporting due to the fact that menomethrorragia is not usually pursued in health surveillance in these workers, further support this hypothesis. The effect might be related to a phlogistic stimulus caused by EMF fields, possibly the low-frequency currents induced in the wires of the IUD during the movement of operators inside the SMF generated by the MRI permanent magnet.

The cases reported here suggest that the possible health effects in MRI Units operators with IUDs deserve more attention, especially considering that more powerful MRI scanners (3 T and more), and interventional magnetic resonance imaging, both potentially involving higher EMF exposure, are increasingly more frequent, and that a significant proportion of MRI operators is composed of females of fertile age, possibly using IUDs. Accordingly, we suggest that the occurrence of menomethrorragia should be looked for in health surveillance of MRI workers. Furthermore, the possibility that MRI operators with implanted metallic IUDs can be included in the group of "workers at particular risk" according to the EU Directive 2004/40/EC [16] should be considered.
More generally, our results support that further research in the field is needed for a more accurate and comprehensive risk assessment, and to effectively protect MRI personnel.

\section{ACKNOWLEDGEMENTS}

Many thanks are due to all the participants to the study, including the Patients that consented to the presentation of the case.

\section{REFERENCES}

1. Hess T, Stepanow B, Knopp MV. Safety of intrauterine contraceptive devices during MR imaging. Eur Radiol 1996;6:66-8.

2. Mühler M, Taupitz M. How safe is magnetic resonance imaging in patients with contraceptive implants? Radiologe 2006;46:574-8.

3. Mishell DR Jr. Intrauterine Devices: Mechanisms of Action, Safety and Efficacy. Contraception 1998;58:45S-53S.

4. Pasquale SA, Russer TJ, Foldesy R, Mezrich RS. Lack of interaction between magnetic resonance imaging and the copperT380A IUD. Contraception 1997;55:169-73.

5. Shellock FG. Radiofrequency Energy-Induced Heating During MR Procedures: A Review. J Magn Reson Imaging 2000;12:30-6.

6. Shellock FG. New Metallic Implant Used for Permanent Contraception in Women: Evaluation of MR Safety. Am J Roentgenol 2002;178:1513-6.

7. Zieman M, Kanal E. Copper T 380A IUD and magnetic resonance imaging. Contraception 2007;75:93-5.

8. Mark AS, Hricak H. Intrauterine contraceptive devices MR imaging. Radiology 1987;162:311-4.

9. ASTM International (American Section of the International Association for Testing Materials). Standard practice for marking medical devices and other items for safety in the magnetic resonance environment. New York: American National Standards Institute; 2005. 
10. Capstick M, McRobbie D, Hand J, Christ A, Kuhn S, Mild KH, et al. An investigation into occupational exposure to electromagnetic fields for personnel working with and around medical magnetic resonance imaging equipment. Report of the Project VT/2007/017. Zurich (CH): Foundation for Research on Information Technology in Society (ITIS); 2008 [cited: 2011 Nov 24]. Available from URL: http://www1.itis. ethz.ch/downloads/VT2007017FinalReportv04.pdf.

11. Keevil SF, Krestin G. EMF Directive still poses a risk to MRI research in Europe. Lancet 2010;376:1124-5.

12. Gallo MF, Nanda K, Grimes DA, Lopez LM, Schulz KF. $20 \mu \mathrm{g}$ versus $>20 \mu \mathrm{g}$ estrogen combined oral contraceptives for contraception. Cochrane Database Syst 2008;Rev 4:CD003989.

13. Ehrmann DA. Polycystic ovary syndrome. N Engl J Med 2005;352:1223-36.
14. Pregler JP, DeCherney AH. Women's Health: Principles and Clinical practice. Hamilton, Ontario: BC Decker Inc.; 2002. 15. Kulier R, O'Brien P, Helmerhorst FM, Usher-Patel M, d'Arcangues C. Copper containing, framed intra-uterine devices for contraception. Cochrane Database Syst 2007;Rev 4:CD005347.

16. EU Directive: Directive 2004/40/EC of the European Parliament and of the Council of 29 April 2004 on the minimum health and safety requirements regarding the exposure of workers to the risks arising from physical agents (electromagnetic fields). Eighteenth individual Directive within the meaning of Article 16(1) of Directive 89/391/EEC. Off J Eur Union 2004;159:1-26.

This work is available in Open Access model and licensed under a Creative Commons Attribution-NonCommercial 3.0 Poland License - http://creativecommons.org/ licenses/by-nc/3.0/pl/deed.en. 